\title{
Das Tao des langen Lebens
}

\author{
The Way of Longevity
}

Autor

Institut

\section{R. M. Trüeb}

Dermatologische Klinik, Universitätsspital Zürich

\section{Bibliografie}

DOI $10.1055 / \mathrm{s}-2008-1077552$

Akt Dermatol 2008; 34:

342-349 ๑ Georg Thieme

Verlag KG Stuttgart · New York ISSN 0340-2541

Korrespondenzadresse

Prof. Dr. Ralph M. Trüeb Dermatologische Klinik Universitätsspital Zürich Gloriastraße 31 8091 Zürich

Schweiz

ralph.trueeb@usz.ch

\section{Zusammenfassung \\ $\nabla$}

Die Medizin zählt zu den ältesten Errungenschaften menschlicher Kultur. Über Jahrtausende hinweg hat sie die Menschheit durch alle Kulturen begleitet, denn sie ist die Antwort auf eine elementare Seinsweise, die Abwehr der unverhofften Unterbrechung des Wohlbefindens, der Peinigung durch körperlichen Schmerz und der Furcht vor dem Tode. Insbesondere das Alter als letzter Lebensabschnitt, seine Gebrechen und der Tod haben Philosophen, Naturwissenschaftler und Ärzte auf den Plan gerufen, nach Zusammenhängen zu forschen, den Alterungserscheinungen, den Alterskrankheiten und dem Tod vorzubeugen. Der Gang durch die Geschichte der europäischen Gesundheitslehre zeigt eine Verflechtung mit Kultur, Religion und Philosophie,

\section{Einleitung \\ $\nabla$}

Die Medizin zählt zu den ältesten Errungenschaften menschlicher Kultur. Über Jahrtausende hinweg hat sie die Menschheit durch die Geschichte begleitet und ihr gedient, denn sie ist eine Antwort auf eine elementare Seinsweise, die der Mensch vom ersten Tage seines Bewusstseins an immer wieder anfechtet, nämlich:

„Die unverhoffte Unterbrechung unseres Wohlbefindens, die Peinigung durch den körperlichen Schmerz und die drohend aufkeimende Furcht vor dem Tode."

(Seneca)

Der appellative Charakter der Schmerz- und Angstäußerung, die nach Beistand ruft, hat die ärztliche Kunst auf den Plan gerufen: Sie ist zu einem fundamentalen Bestandteil aller menschlichen Kulturen zu allen Zeiten geworden. Der Gang durch die Geschichte der europäischen Gesundheitslehre zeigt eine enge Verflechtung mit Kultur, Religion und Philosophie, bis sich im bis sich im 19. Jh. die Diätetik auf Diät, die Hygiene auf Technik und der Gesundheitsbegriff auf messbare Größen reduzierte, während Grundsätze wie Regelmäßigkeit der Lebensgestaltung, Maßhalten, Entspannung und Harmoniestreben in den Hintergrund gerieten. Meditation, Entspannungsübungen, der Wechsel von Ruhe und Bewegung, ästhetische Erholung und religiöse Entrückung sind demgegenüber bis heute Bestandteile der asiatischen Heilkunde geblieben, des Ayurweda und der traditionellen chinesischen Medizin. Die den asiatischen Heilmethoden zugeschriebenen Effekte erklären sich durch außergewöhnliche psychophysiologische Auswirkungen, die die moderne medizinische Forschung und Anti-Aging-Medizin erst wieder zu achten lernen.

19. Jh. die Diätetik auf Diät, die Hygiene auf Technik und der Gesundheitsbegriff auf messbare Größen reduzierte [1]. Bei aller Hochachtung für die Errungenschaften der modernen, naturwissenschaftlichen Medizin, ist es aber bedauerlich, dass einfache Grundsätze wie Regelmäßigkeit der Lebensgestaltung, Maßhalten, Entspannung und Harmoniestreben in den Hintergrund getreten sind. Meditation, Entspannungsübungen, der Wechsel von Ruhe und Bewegung, ästhetische Erholung und religiöse Entrückung, die bis heute Bestandteile der asiatischen Heilkunde sind, wurden von der „messenden“ Medizin verdrängt, obwohl sie genauso selbstverständlich zu unserer Medizingeschichte gehörten, wie die seit Rudolf Virchow („Über das Bedürfnis und die Richtigkeit einer Medizin vom mechanischen Standpunkt“, Rede gehalten 1845) dominierenden rationalen Wege von Prophylaxe und Therapie [2-11]. 


\section{Ayurweda}

$\nabla$

Unsere Kenntnisse der alten indischen Medizin verdanken wir umfangreichen Textsammlungen, wovon die wichtigsten die Samhita oder „Sammlungen“ darstellen, die den Autoren Charak und Sushruta zugeschrieben werden. Sie stellen das medizinische Wissen einerseits als göttliche Offenbarung in Form von Legenden dar, andererseits als „Wissen über die Lebenserwartung“, das ein Teilgebiet der Weden ausmacht, die mit einem Alter von über 3000 Jahren zu den ältesten klassischen Schriften der Welt gehören. Bei den Weden handelt es sich um Sammlungen von Hymnen, liturgischen Gesängen, Opfersprüchen und Zauberformeln. Entsprechend beruhte die wedische Therapie ihrem Wesen nach zunächst auf heilkräftige Beschwörungsformeln und magischen Praktiken. Viele der wedischen Götter erscheinen als Heilende, und in zahlreichen Hymnen werden sie um Gesundheit und ein langes Leben angerufen. Zuoberst in der Hierarchie dieser Götter gehören die Aswin-Zwillinge, die als göttliche Ärzte galten. Preisgesänge erinnern daran, dass sie altersschwachen Greisen die Jugend wiedergaben:

„Ihr habt Tschjawana die Kraft der Jugend wiedergeschenkt.“

(Rigweda, I, 118, 6-7)

Die Überlieferung hat auch aus Indra aufgrund seiner Beziehungen zu den Aswin einen Gott der Medizin gemacht. Der Sage um den Ursprung der indischen Medizin nach, soll er dem ersten einer Reihe von Ärzten die medizinischen Kenntnisse der Aswin übermittelt haben. Einige Hymnen des Rigweda (I; 165, 15; 169, $8 ; 176,6)$ erbitten von ihm ein langes Leben in Wohlstand. Ein weiterer wedischer Gott und Vorläufer des hinduistischen Schiwa, Rudra, gilt ebenfalls als Wunderheiler und Arzt:

„Dank deiner heilkräftigen Medikamente, o Rudra, möge ich einhundert Winter erleben!“

(Rigweda, II, 33, 2-3)

Allerdings handelte es sich um einen Gott, den man mit Vorsicht anrufen musste, denn er galt als reizbar, und sein Zorn verwandelte ihn dann in eine zerstörende Gottheit, die den Menschen mit Leid und Tod belegte. Aber als Verursacher von Krankheit war er auch am ehesten in der Lage, diese zu heilen. Schließlich ist die Gottheit Waruna zu erwähnen, der als Gott der Gewässer erscheint. In den Weden bedingen die Wasser den Gang des Universums sowie das Leben der Geschöpfe, weshalb in den Wassern alle Heilmittel angenommen wurden (Rigweda, X, 9,6), auch die Flüssigkeit der Unsterblichkeit (Rigweda, I, 23, 19), als deren Hüter Waruna angesehen wurde (Rigweda, VIII, 42, 2).

Man hat aber auch die Wirkung bestimmter Pflanzen auf den Organismus beobachtet. Durch die Notwendigkeit, für die tägliche Nahrung zu sorgen, dazu getrieben, in der Umgebung wachsende Kräuter kennen zu lernen, hat man zweifelsohne im Verlauf der Generationen die Heilpflanzen entdeckt. Der Gebrauch pflanzlicher Drogen zu therapeutischen Zwecken ist belegt durch den Heilkräutern gewidmeten Passagen des Atharweda (VII,7):

„Wir rufen alle Pflanzen an, braune und weisse, rote und buntscheckige, dunkle und schwarze. Mögen diese Kräuter jenen Menschen dort von dem Leiden befreien, das ihm von den Göttern auferlegt wurde."

Als „König der Heilpflanzen“ galt der Soma, dem die einhundertzwanzig Hymnen des IX. Buches des Rigweda gewidmet sind. Er wächst in den Bergen und wird von einem Vogel gebracht. Man glaubte, dass der aus dem Soma gepresste Saft die Gedanken stimuliert, Mut macht, die Potenz fördert und Krankheit heilt. Er wurde von den Priestern und Gott getrunken und verlieh ein Ge- meinschaftsgefühl mit der Gottheit und Sicherheit des Nicht-Todes. Wer das Soma-Opfer darbrachte, wurde 100 Jahre alt, was der Unsterblichkeit gleichkommt (Rigweda, VIII, 48,3; IX, 103, 3):

„Wir haben Soma getrunken, unsterblich sind wir geworden, gekommen sind wir zum Licht, aufgefunden haben wir die Götter. Was könnte uns jetzt noch Missgunst antun, was, o Unsterblicher, die List eines Sterblichen? Sei uns getrunken, Segen im Herzen, o Tropfen, freundlich, o Soma, wie der Vater zum Sohn, wie der Freund als weithin herrschender Weiser. Unsere Lebenskraft verlängere zum vollen Leben, o Soma!“

„Mit munterem Sinne wollen wir von deinem ausgepresstem Saft geniessen wie den väterlichen Reichtum. O König Soma, verlängere unsere Lebensdauer wie die morgendlichen Tage!"

„Dem gütigen Freunde will ich folgen, der mich als Getrunkener nicht schädigen möge; du falbe Rosse Besitzender! Der in uns enthaltene Soma, für diesen gehe ich zu Indra (mit der Bitte) um Verlängerung des Lebens. Hinweggegangen sind jetzt Entkräftung und Krankheit, entflohen sind die Ermattenden; sie fürchten sich. Für uns ist der Soma aufgestiegen gewaltig; wir sind dorthin gegangen, wo man das Leben verlängert. Der als Tropfen, der o Ahnen, getrunken Eingang gefunden hat in unsere Herzen als Unsterblicher in Sterbliche, diesem Soma wollen wir mit dem Opfergut huldigen. In seiner Gnade und Wohlgesinntheit möchten wir uns befinden. Du, o Soma, bist mit den Ahnen vereint; ausgedehnt hast du dich wie Himmel und Erde. Darum wollen wir dir, o Tropfen, mit Opfergut huldigen. Wir wollen die Herren der Reichtümer sein."

Abermals ist versucht worden, diese Pflanze zu identifizieren, aber mehrere Gewächse konnten zu verschiedenen Zeiten und in verschiedenen geografischen Regionen denselben Namen tragen, sodass bald die Frage aufgeworfen wurde, ob es sich nicht um eine mythische Pflanze handle. Dennoch, die zahlreichen pflanzlichen Produkte, die die wedische Medizin einsetzte, bieten ungeahnte Möglichkeiten diese auf pharmakologisch aktive Substanzen zu identifizieren. Beispiele sind die SarpagandhaPflanze (Rauwolfia serpentina), die den Bluthochdruck senkt, und Commiphora wightti-Harz, der erhöhte Blutfette senkt. Entsprechend werden heute in Forschungslaboratorien in Indien Arzneipflanzen der wedischen Medizin systematisch auf ihre pharmakologische Wirkungen untersucht.

Erst gegen Ende der wedischen Zeit flossen die empirischen und rationalen Elemente in die indische Medizin ein, die die Grundlage zur zusammenhängenden Lehre des Ajurweda bilden, dem „Wissen über das lange Leben“. Die ayurwedische Therapeutik wurde nicht allein von der aufgrund ihrer Symptomatik diagnostizierten Krankheit bestimmt, sondern auch von deren Ursachen. Größtenteils verfuhr man ätiologisch, wobei falsche Ernährung und falsches Verhalten nach Ansicht der indischen Ärzte in beträchtlichem Ausmaß zur Entstehung von Krankheiten beitragen. Daher räumt die ayurwedische Medizin den diätetischen Maßnahmen und Hygienevorschriften einen wichtigen Platz ein. Altgriechische Quellen aus der Zeit Alexanders des Großen (356- 323 v. Chr.), der auf seinem Siegeszug durch Asien bis nach Indien drang, ermöglichen es heute, eine Vorstellung von den damaligen Fortschritten der indischen Medizin zu gewinnen. Onesikritos von Astypalaia berichtete zu jener Zeit, dass die Inder ihr langes Leben einer vollkommenen Körperpflege verdankten.

Die unter dem Begriff Tantrismus bekannte zeitgenössische Mystik begünstigte die Aufnahme auch psycho-physiologischer Methoden, die vom Yoga inspiriert waren, in das Ayurweda. Besonders die Atemlehre des Yoga (Hathayoga) lehrt, dass bestimmte 
Atemübungen das sicherste Mittel seien, um Krankheiten, dem Altern und dem Tod zu entgehen. Diese Vorstellungen beruhten auf der Auffassung der wedischen Autoren über die Funktion des Organismus in Verbindung mit dem Wind, welche die Seele der Welt sei, jene kosmische Kraft, die sowohl den Makro- als auch den Mikrokosmos belebe. Die gesamte Physiologie des Mikrokosmos beruht demnach auf dem Kreislauf der „Lebensluft“, die durch entsprechende Atemübungen im Organismus richtig zu kanalisieren sei. Die dieser Praxis zugeschriebenen Effekte erklären sich heute durch ihre außergewöhnlichen psychophysiologischen Auswirkungen, die die medizinische Forschung in den letzten Jahrzehnten zu achten gelernt hat. Dabei handelt es sich besonders um Wirkungen auf die Herz- und Atemfunktionen. Gewisse Yoga-Übungen führen zu einer erheblichen Senkung des Sauerstoffverbrauchs und des Pulsschlags, Einflüsse die aus heutiger Sicht zum längeren Leben beitragen.

\section{Traditionelle chinesische Medizin \\ $\nabla$}

Im alten China wurden seit Urzeiten Geschichten von Menschen, die ein langes Leben, ja Unsterblichkeit erreichten, überliefert und studiert. Chi Kang, einer der Sieben Weisen vom Bambushain, arbeitete während der Chin-Dynastie eine Theorie der Lebensführung aus, mit der er versuchte, die Wahrheit der Theorie der Unsterblichkeit zu beweisen. Er argumentierte, dass es förderlich sei, zwecks Erreichung eines langen Lebens eine geregelte Lebensweise einzuhalten, und dass der Weg (Tao) der Unsterblichkeit durch Überwindung der Weltlichkeit die Erlangung der Göttlichkeit ermögliche. Obwohl bis heute die Behauptung Chi Kangs, der zufolge der Weg der Unsterblichkeit über die spirituelle Transzendenz führe, weder bestätigt noch widerlegt wurde, kann eine Theorie, die über Jahrtausende von Generation zu Generation überliefert und mitunter auch praktiziert wurde, nicht umhin, eine gewisse Legitimität zu beanspruchen, wenn auch in einer anderen, nämlich einer mystischen Dimension.

Die alt-chinesische Auffassung von Gesundheit und Krankheit beruht auf über zweitausendjährige Erfahrung und beansprucht eine holistische Betrachtungsweise des menschlichen Organismus und seiner Störungen. Der Mensch, der seinen Platz zwischen Himmel und Erde hat, muss sich in der äußeren Welt in das Spannungsfeld der gegensätzlichen Kräfte von Yin und Yang einfügen. Dessen Ungleichgewicht bildet die Grundlage für Erkrankung. Aus diesem Grund ist die chinesische Medizin innig mit der Kosmologie verbunden und neigt dazu, das Betätigungsfeld des Praktikers zu erweitern und mit dem Universum zu vereinen.

Die traditionelle chinesische Medizin (TCM) gründet auf überkommene Vorschriften, die im Laufe von Jahrhunderten entwickelt wurden, sich letztlich aber auf den Mythos berufen. Die Bezugnahme auf Vergangenheit und Tradition bildet einerseits einen Festpunkt, dokumentiert andererseits aber auch die fortlaufende Entwicklung einer Gesellschaftsordnung. Die Biografien und Ikonografie vorbildlicher Kaiser einer sagenhaften Vorzeit sind Kreationen aus der Erinnerung chinesischer Schriftsteller. Die legendären Kaiser waren nicht nur Musterbeispiele guter Herrscher, sondern galten auch als Vorbilder auf dem Gebiet der Medizin. $\mathrm{Zu}$ den legendären Begründern der Heilkunst des Fernen Ostens zählen die sagenhaften Kaiser Fu-hi, Schen-nong und Huang-ti ( $\bullet$ Abb. 1).

Fu-hi soll das Abkochen der Nahrung erfunden haben, und ihm wurde retrospektiv die Zusammenstellung des „Buchs der

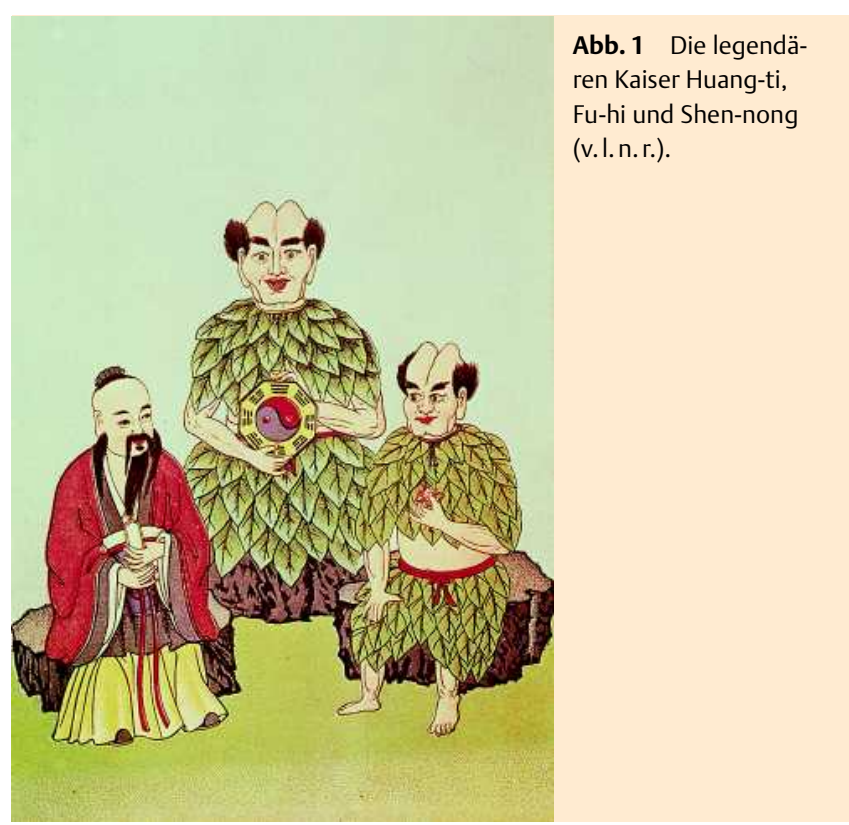

Wandlungen“ (I Ging) zugeschrieben. Die Überlieferung berichtet, dass er eines Tages ein Drachenpferd dem Gelben Fluss entsteigen sah. Er erkannte die acht Diagramme auf dem Rücken des Tieres und bestimmte das Wesen der Verwandlungen: Ihre zeichnerische Darstellung, die aus übereinander gelagerten, durchbrochenen (Yin) oder durchgezogenen (Yang) Strichen bestehen, geben Einblick in die Prinzipien des Yin und Yang und ihre Wechselwirkungen. Die Vielfalt der Bedeutungen, welche die Trigramme in ihren unterschiedlichen Kombinationen abdecken können, gibt dem um eine Klassifizierung der Krankheiten bemühte Arzt eine Lösungsmöglichkeit. Die Lehre des Fu-hi hat den Grundstein zu einer Naturphilosophie gelegt, aus der abgeleitet wird, dass die Veränderung der Gesundheit des Menschen einen Bruch mit der kosmischen Ordnung „T'ien-ti“ bedeutet. Schen-nong wird die Erfindung der Arzneimittellehre zugeschrieben: Er soll hundert Pflanzen geprüft haben und die „Klassische Abhandlung über die materia medica“ (Pen-ts'ao king) zusammengestellt haben. Das Pen-ts'ao king umfasst 365 Produkte tierischen, pflanzlichen und mineralischen Ursprungs und fügt sich in die Kosmologie ein. Es werden die „höheren“, die „mittleren“ und die „niederen Arzneimittel“ unterschieden: Die 120 „höheren Arzneimittel“ sind unabhängig von der Dosierung niemals giftig. Sie sind dazu bestimmt, „das Leben zu nähren“ und gegen den Alterungsprozess anzukämpfen. Beispiel ist das Jenshen oder die Ginsengwurzel (Radix Ginseng). Die 120 „mittleren Arzneimittel“ sind manchmal giftig und können Unverträglichkeitsreaktionen hervorrufen. Sie sollen „das Lebensprinzip nähren“ und Mangelerscheinungen beheben. Die 125 verschiedenen „niederen Arzneimittel“ werden schließlich mit Vorsicht verordnet und zur Behandlung von Krankheiten eingesetzt. In Wahrheit wurde das Pen-ts'ao king während der Han-Dynastie zwischen 32 v. Chr. und $10 \mathrm{n}$. Chr. von einer Gruppe unbekannter Mediziner zusammengestellt, die den Namen des Schen-nong für ihre Sache nutzten. Die Vorschriften geben ein Bild der altchinesischen ärztlichen Mentalität: Sie sind eine Anleitung für ein langes Leben durch materielle Erhaltung des Körpers, wenngleich die von diesen Wertvorstellungen durchdrungenen, korrumpierten taoistischen Mediziner und Scharlatane späterer Jahrhunderte auch nach der „Unsterblichkeitsdroge“ geforscht haben. 


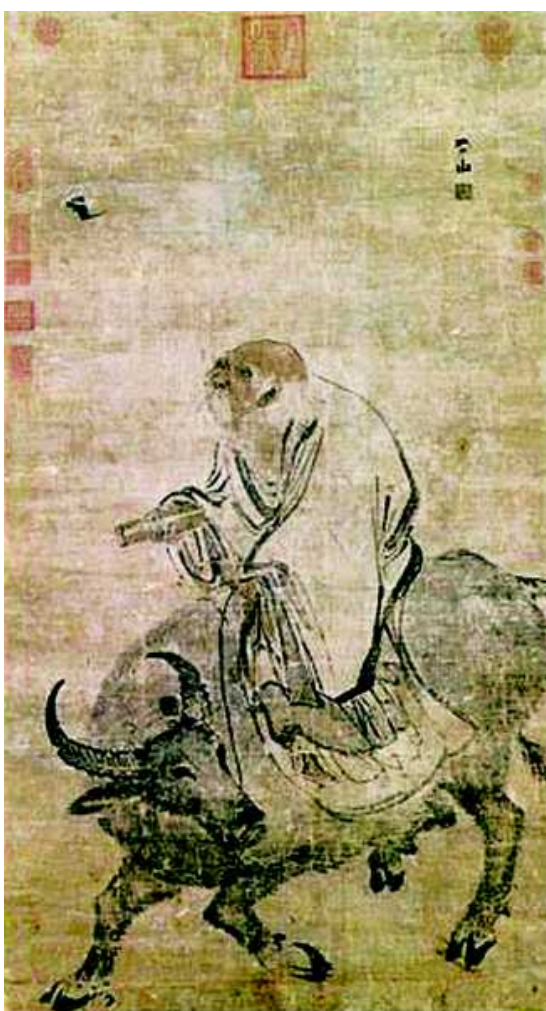

Abb. 2 Lao-tzu.

Huang-ti, dem „Gelben Kaiser“, schreibt die Überlieferung die Abfassung des Huang-ti Nei-king su-wen („Grundlegenden Probleme des klassischen Lehrbuchs des Huang-ti über die Innere Medizin“) zu, das eine Abhandlung von Grundfragen der Anatomie, Physiologie, Pathologie und Therapie im chinesichen Altertum (Su-Wen) und ein „Traktat über die Akupunktur“ (Ling-schu) umfasst. Es präsentiert sich in der Form von Zwiegesprächen zwischen dem legendären „Gelben Kaiser“ und seinen ärztlichen Ratgebern. Das Nei-king stellt eine Quelle der chinesischen Medizin dar, aus der bis heute traditionell orientierte Praktiker ihren Wissensschatz schöpfen. Obwohl der mythische Herrscher Huang-ti in die Zeit um 2000 v. Chr. eingeordnet wird, liegen wiederum genügend medizinhistorische Fakten vor, um anzunehmen, dass das Nei-King erst gegen Ende der späteren ChouHerrschaft, in der Zeit der Kämpfenden Reiche (475 - 221 v. Chr.) oder sogar etwas später zusammengestellt wurde. Nach der Überlieferung soll der „Gelbe Kaiser“ die meiste Zeit seines Lebens damit verbracht haben, dem Geheimnis des ewigen Lebens auf die Spur zu kommen. Sein Interesse an der Behandlung von Krankheiten, der Stärkung der Lebenskraft und der Verlängerung der normalen Lebensdauer, hatte ihn dazu geführt sich mit den Praktiken zur Erlangung von Unsterblichkeit zu beschäftigen. Er führte alchimistische Experimente durch und soll mit Erfolg ein „Goldenes Elixier“ destilliert haben. Als diese Droge zu wirken begann, verwandelte er sich, „bestieg einen Drachen und flog davon, zum Reich der Unsterblichen“. Kurz zuvor hatte er die Formel an Personen weitergegeben, die sie aber in ihrer Unachtsamkeit verloren! Heute lässt sich aber nicht mehr genau bestimmen, wonach Huang-ti suchte, denn der Taoismus, wonach alles Existierende aus Geist besteht, unterschieden in ihren frühen Schriften nicht zwischen Geist und Materie.

Die Epoche, welcher die legendären Kaiser angehörten und die nach chinesischer Auffassung vor nahezu 5000 Jahren begann, liegt zu weit zurück, als dass sie historisch enger gefasst werden könnte. Die Übereinstimmungen, die zwischen den Mythen und den mystischen Lehren des Altertums bestehen, haben zu einer Hypothese geführt, nach der es auf der ganzen Welt tatsächlich ein „Goldenes Zeitalter“ gegeben habe, in der Götter und Menschen innerhalb einer Hochkultur einander näher standen als jemals wieder. Über den Untergang dieser Hochkultur ist nichts bekannt. Der Untergang muss während einer jener Weltkatastrophen stattgefunden haben, mit denen die dialektische Natur einen Ausgleich zwischen Fortschritt und Umkehr schafft. Nur wenige verstreute Reste in Form von rätselhaften Bruchstücken uralter Weisheiten sollen davon übrig geblieben sein. Eine Vorstellung ist, dass der Taoismus etwas von dieser untergegangenen Weisheit bewahrt haben soll. Lao-Tzu ( Abb. 2 angeblich 6. Jh. v. Chr.) gilt als Verfasser des Tao Te Ching („Buch vom Weg und seiner Wirkung“), obwohl die neuere Forschung das Werk für anonym und diese Zuschreibung für fiktiv hält. Die „Biografie" des Lao-Tzu, wie sie beim chinesischen Historiker Ssu-ma Ch'ien (135-87 v. Chr.) steht, erzählt die wohlbekannte Legende vom königlichen Archivar, der sich, der Welt überdrüssig, nach dem fernen Westen aufmacht und am Grenzübergang, vom Passhüter um Hinterlassung seiner Weisheit für kommende Generationen gebeten, „ein Buch in zwei Teilen von mehr als fünftausend Worten“ niederschreibt. Über sein Ende gibt es unterschiedliche Angaben. Einige berichten, dass er 160 Jahre alt wurde, andere, dass er über 200 Jahre alt wurde. Mit der Zeit wurde Lao-Tzu zur völlig legendären Gestalt mit göttlichen Zügen, die zum Teil der Buddha-Legende entlehnt sind, und galt als Begründer des Taoismus. Der Ursprung jener Lebensweisheit, als deren Vater Lao-Tzu fortan galt, liegt allerdings im Dunkeln. Ihr Zentralbegriff, das Tao, könnte im ältesten Taoismus als „Weg zum ewigen Leben“ verstanden worden sein. In einer Ausweitung des Begriffs wurde Tao sodann zum Weg der Natur, zum Urgrund allen Seins und schließlich zum Namen des Unbenennbaren schlechthin. Ziel der taoistischen Mystik war es, zu diesem Weg der ursprünglichen Natur und damit zu dauerndem Leben heimzukehren. Als Hindernisse sind Eigensucht und Gewinnstreben zu verwerfen. Nachdem unweigerlich auf die Blüte das Welken folgt, kann Glück nur durch ein Aufhalten des Erfolges, und Leben nur durch ein Aufhalten des Alterns gewonnen werden. Im Unterschied zur griechischen Philosophie und zum christlichen Glauben, die Diesseits und Jenseits klar trennen, sieht der Taoist das ewige Leben als eine Fortsetzung der leiblichen Existenz an. Nach einer späteren Auffassung endet die Möglichkeit, unsterblich zu werden, mit dem siebzigsten Lebensjahr, der Idealzustand ist der des „Kindleins“:

„Die Völligkeit dessen, der Tugend in sich versammelt,

Gleicht der eines neugeborenen Kindleins.

Bienen, Skorpione, Vipern und Schlangen beissen es nicht;

Wilde Tieren schlagen es nicht,

Raubvögel reissen es nicht.

Seine Knochen sind schwach, seine Sehnen weich,

Dennoch ist fest sein Griff.

Es weiss noch nicht von Mannes und Weibes Vereinigung,

Dennoch zeigt sich sein Glied:

Das ist der Samenkraft Gipfel.

Den ganzen Tag schreit es,

Dennoch wird es nicht heiser:

Das ist der Gipfel natürlichen Einklangs.

Den Einklang kennen heisst: Ewig sein.

Das Ewige kennen heisst: Erleuchtet sein.

Das Leben mehren heisst: Unheil beschwören.

Bewusst den Atem regeln heisst: Stärke begehren. 
Wird ein Wesen fest, so wird es alt.

Dieses nennt man: Nicht dem Weg gemäss.

Nicht dem Weg gemäss wird enden bald.“

Erreicht wird dieser selige Zustand durch „Beschränkung der Samen, Worte und Taten“. Wu-wei („ohne Tun“) ist einer der beliebtesten Termini sämtlicher taoistischer Schriften. Wu-wei war einer der Hauptfaktoren für den Erfolg taoistischer Adepten, ihre geistigen und körperlichen Kräfte bis ins fortgeschrittene Alter zu bewahren und ein langes Leben zu genießen. Wo Sorge und Berechnung fehlen, entsteht auch wenig Verschleiß.

„Menschen von begrenzten Gaben zwingen ihren Geist, und dadurch schaden sie sich nur selbst. Wenn die Zurückhaltung beim Kräfteeinsatz durch Zwang ersetzt wird, ist das Ergebnis nicht gut. Sorge und Melancholie sind schädlich. Ein zorniges Gemüt ist schädlich. Übermässige Zuneigung zu geliebten Menschen ist schädlich. Die Zeit mit müssigem Schwatzen und Scherzen zu verbringen ist schädlich. Ständig Sport zu treiben ist schädlich. Übermässig zu trinken und zu essen, dass man ganz träge wird, ist schädlich. Hin und her zu hetzen, bis man keucht, etwas mit solchem Eifer zu tun, dass die Gelassenheit verloren geht, den Missmut nicht unter Kontrolle zu halten, lachen, bis die Tränen in die Augen steigen - all diese Störungen des Gleichgewichts von yin und yang sind schädlich. Wer es zulässt, dass sich derlei Schäden über die Jahre hinweg anhäufen, stirbt jung.“

Der Einfluss des Taoismus führte zur Empfehlung einer Askese insbesondere für die alten Tage. Da lediglich die Unsterblichkeit des Körpers angestrebt werden konnte, versuchte man, diesem seine Leistungsfähigkeit voll zu erhalten, indem man vermied, seine Lebensenergie durch heftige Leidenschaften, Sinnenfreuden und Ejakulationen zu verschwenden.

Mao Meng (3. Jh. v. Chr.) ist der Erste unter den taoistischen Weisen der Frühzeit, von dem einige Lebensdaten bekannt sind. $\mathrm{Er}$ und eine lange Reihe von Meistern, die ihm folgten, waren auf unterschiedliche Weise darum bemüht, Unsterblichkeit zu erlangen. Sie nahmen an, das Leben könne durch sorgfältige Schutzmaßnahmen für den Körper, wie Diät, Bewegungsübungen (T'ai chi chüan) und sexuelle Beherrschung und richtige Atemtechnik bis zur Erlangung der Unsterblichkeit verlängert werden. Zu den einschlägigen Praktiken gehörten: das Einhalten einer strengen Diät; das Baden zu vorgeschriebenen Zeiten und in Übereinstimmung mit einem in allen Einzelheiten festgelegten Zeremoniell; das Mischen von Elixieren, von denen einige gefährliche Zutaten enthielten; der rituelle Geschlechtsverkehr unter Beachtung von Anweisungen bezüglich Zeitplanung, Position, Rhythmus usw. (sog. „wechselseitige Kultivierung“); verschiedene Atemtechniken; die Meditation und der Gebrauch von Amuletten.

Taoistische Begriffe wie „Goldenes Elixier“ und „Veredlung“ verweisen richtig auf die psychophysischen Prozesse taoistischer Meditationspraktiken, sind aber nur allzu oft missverstanden worden. Über Jahrhunderte wurde gemeinhin angenommen, dass taoistische Alchimisten tatsächlich eine Droge herstellen könnten, die ewige Jugend und Unsterblichkeit verleihe. Nach diesem Glauben wurde der Körper eines erfolgreichen Adepten in eine gewichtlose, Jade-ähnliche Substanz umgewandelt, die unempfindlich gegen extreme Hitze oder Kälte war, von einem Häppchen Wind und einem Schlückchen Tau genährt werden konnte und so fähig war, für immer als „Unsterblicher“ zu bestehen. Man stellte sich vor, dass solche Wesen in den unzugänglichen Bergen Zentralasiens oder auf den märchenhaften P'engLai-Inseln im Ostmeer wohnten.
Im Jahre $221 \mathrm{v}$. Chr. erzwang der König von Tsin, Yin Tschen (259-210 v. Chr.), die Einheit Chinas, nachdem er die sechs einander befehdenden Staaten Han, Tschao, Wie, Tch'u, Yen und Ts'i unterwarf, und setzte damit der Zeit der einander bekämpfenden Einzelstaaten ein Ende. Er proklamierte sich selbst zum Kaiser und Begründer der Tsin-Dynastie (221 - 206 v. Chr.), Tsin Sche Huang-ti. Tsin Sche Huang-ti schuf einen Zentralstaat, sorgte selbst für die Vereinheitlichung der Sprache, Währung, der Maße und Gewichte, der Straßenbreiten und selbst des Radstandes an den Wagen. Aufseher kontrollierten die Arbeit der Verwaltungsbeamten, und ihre Berichte gingen direkt an den Kaiser. Am Kaiserhof drehte sich indessen alles um die Suche nach der „Droge des langen Lebens“. Nach der Einigung des Reiches verwandte Sche Huang-ti seine ganze Energie auf die vergebliche Suche nach dem Elixier für ein langes Leben, im Bestreben seiner zentralistischen Vorstellung von der kaiserlichen Gewalt auch Zeit und Raum unterzuordnen. Sche Huang-ti war nur einer von mehreren Herrschern, die von der esoterischen Sprache der taoistischen Schriften zu der Hoffnung verleitet wurde, die leibliche Unsterblichkeit zu erlangen. Sche Huang-ti umgab sich mit einem Hofstaat von Schwarzkünstlern, und war - genauso wie nach ihm ein anderer großer Kaiser, Han Wu Ti (140-88 v. Chr.) - darauf versessen, den Aufenthaltsort der „Unsterblichen“ mit eigenen Augen zu sehen, rüstete er kostspielige Expeditionen zu den Inseln vor der Küste Shantungs. Die Mediziner unterschieden sich kaum von den Zauberkünstlern. Besonderes Ansehen kam dem Oberarzt des Frauengemaches zu. Die Suche nach Methoden zur Verlängerung des Lebens, führte dazu, Störungen des Gleichgewichts von Ying und Yang mit größter Sorgfalt zu beobachten. Unregelmäßigkeiten des Menstruationszyklus wurden genau registriert, und man erkannte, dass das endgültige Ausbleiben der Regelblutung den Beginn jenes Zeitraumes markiert, in dem die Frau keine Kinder mehr gebären kann. Mit 49 Jahren stellt das „Gefäß der Empfängnis“ seine Tätigkeit ein, und die Menopause signalisiert den Verfall des T'ai-tschong, des „Lebensgefässes“, das seine Funktionen immer weniger wahrnimmt. Der Kampf gegen den Alterungsprozess traf mit der Suche nach der Droge für ein langes Leben zusammen. Während der Tsin-Dynastie wurde insbesondere den kryptogamen Pflanzen (Pilze, Farne, Moos) wundersame Eigenschaften zugeschrieben. Die berühmteste war der göttliche Ling-tsche oder Ganoderma japonicus (Fr.) Lloyd.

In der Zeit der Östlichen Han-Dynastie (25-220 n. Chr.) verfasste Chang Tao-ling ein Talisman-Buch über zauberkräftige Gegenstände zur Heilung von Kranken. Chang hatte ursprünglich die Stelle eines Armeesekretärs inne. Als seine Einheit von einer Epidemie dezimiert wurde, schrieb er sein Überleben der Kraft eines Amuletts zu, das er zum Bannen vom Dämonen bei sich trug. Darauf entschloss er sich, den Rest seines Lebens mit der Kultivierung des Tao zu verbringen. Sein Interesse wuchs jedoch rasch über die Geisterbeschwörung hinaus, und bald verfasste er Bücher über Hygiene und Heilkunst. Seine bemerkenswerten Heilmethoden erregten soviel Aufmerksamkeit, dass ihn zwei Kaiser nacheinander an ihren Hof rufen ließen. In wahrhaft taoistischer Art wies er aber diese Einladungen zurück und begab sich in die Einsamkeit der Berge, wo er in seinem 123. Lebensjahr „einen Drachen bestieg und auf in das Reich der Unsterblichen fuhr“. Chang war der erste einer Reihe von „Meister des Himmels“, T'ien-sche, die zum offiziellen Haupt des Taoismus wurden. Seine spirituellen Nachkommen bekleideten dieses Amt nahezu 2000 Jahre lang, bevor der letzte von ihnen 1949 von den Kommunisten vertrieben wurde. Als ebenfalls hoch ver- 


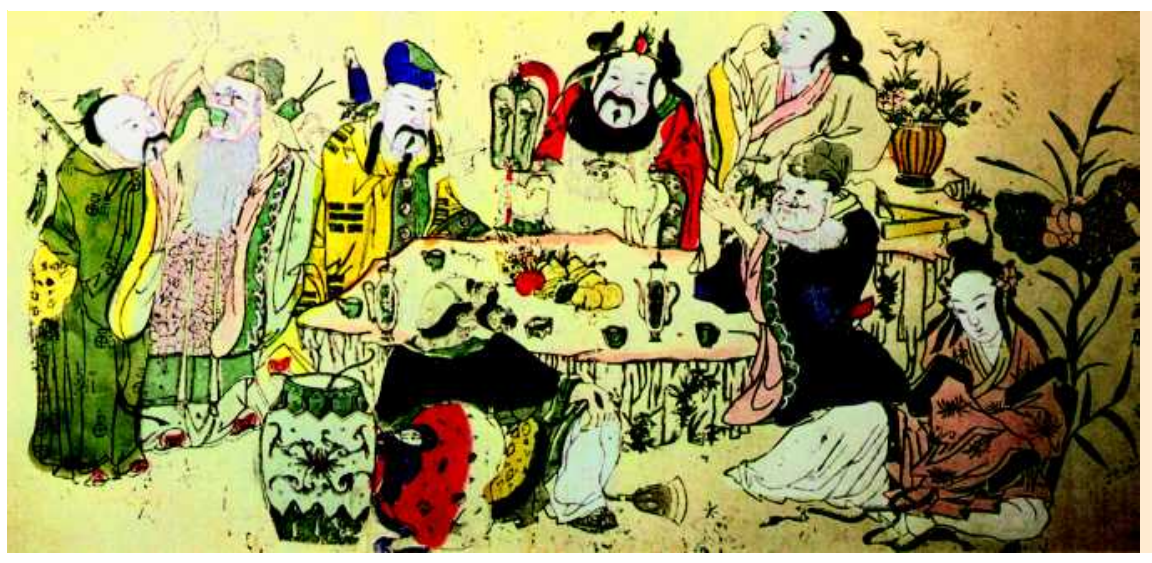

Abb. 3 Die „Acht Unsterblichen“.

ehrter taoistischer Meister gilt Wei Po-Yang (2. Jh. n. Chr.), der mit seinem außergewöhnlichen Buch Ts'an T'ung Ch'i („Dreifacher Einklang“') ein esoterisches Handbuch liefert, das einen entscheidenden Beitrag zur Entwicklung des Taoismus leistete. Das Werk zeichnet sich durch eine derart rätselhafte Ausdrucksweise aus, dass es ungeklärt bleibt, ob manche Passagen wie die Anweisungen, nichtedle Metalle in Gold zu verwandeln oder eine „Goldpille“ zu mischen, die Unsterblichkeit verleiht, wörtlich zu nehmen sind.

Als einer der größten taoistischen Meister aller Zeiten wird Ko Hung (253-334 n. Chr.) angesehen, der unter dem Beinamen Pao P'u Tzu („Der Meister, der am Einfachen festhält“), dem Titel seines um 317 entstandenen Werkes weitaus bekannter ist. Ko Hung stammte aus einer konfuzianischen Familie gelehrter Beamter und hatte von frühester Jugend an viel Freude am Lernen. Er erwarb eine umfassende Bildung, und für den Eintritt in die Beamtenlaufbahn und für einen hohen Rang, wie ihn seine Vorfahren erreicht hatten, studierte er die „Vier Bücher“ und „Fünf Klassiker" so gründlich, dass ihn die Konfuzianer als einen Eingeweihten auf ihrem eigenen Wissensgebiet verehrten. Dennoch fühlte er sich zur taoistischen Mystik hingezogen, und verfolgte seine Interessen in dieser Richtung. Während der Regierungzeit des Kaisers Hui Ti (290-307 n. Chr.) erhielt er den Oberbefehl über die Kaiserlichen Truppen, aber trotz seines Erfolges hinterließ diese Erfahrung bei Ko Hung derart eine Abneigung gegen das öffentliche Leben, das er sich entschloss, in ländlicher Abgeschiedenheit Zuflucht vor der Welt des Staubes zu suchen. Schließlich ließ er sich auf dem Lo-Fu-Berg nieder, wo er sich in die Kultivierung des Tao vertiefte. Als er mit 81 Jahren starb, fand man ihn in Meditationshaltung, und sein Aussehen soll noch außergewöhnliche Jugendfrische besessen haben. Pao P'u Tzu verfasste nicht weniger als 116 Bände, und seine philosophischen Schriften waren von einem kritischen taoistischen Geist erfüllt: „Gewöhnliche Menschen, die nach Ruhm und Reichtümer streben, können nicht damit rechnen, zum Tao zu gelangen, weil dafür äusserste Entschlossenheit erforderlich ist. Langlebigkeit oder Unsterblichkeit zu gewinnen, ist keine Sache, die man durch Rituale, das Aufsagen von Zaubersprüchen oder das Einnehmen von Zaubertränken erreicht; als oberste Erfordernis gilt es, tugendhaft zu sein uns sich mit Festigkeit vom Bösen fernzuhalten. Taoisten, die nur darauf bedacht sind, durch alchimistische Techniken ihren Körper zu stärken, sind zum Scheitern verurteilt.“

Möglicherweise gelang Pao P'u Tzu erst spät zu den oben zitierten Ansichten, denn einige seiner früheren Schriften befassen sich eher mit weltlichen Angelegenheiten. Er verschreibt zum Beispiel zum Zweck der Verjüngung, Geisteskraft, Kälteunemp- findlichkeit, Nachtsichtigkeit, Körperkraft und zu einer Lebensdauer von mehr als hundert Jahren Pflanzenpräparate auf Basis von Atactylis ovata, Acorus calamus, Rehmannia glutinosa und Asparagus lucidus. In diesen Präparaten soll man Gold, Quecksilber, Jade, Schwefel, Zinnober, Auripigment, Quarz und Blei auflösen. Außerdem legte er großen Wert auf Atemübungen, die er als Mittel zur Vorbeugung gegen Krankheiten verstand, sowie zum Gewinn übernatürlicher Kräfte. In seinen „Esoterischen Schriften“, behandelt Pao P'u Tzu eine verwirrende Vielzahl von Themen und so schwer verständliche Dinge wie die geheimnisvollen Gesetze des Universums, das Tao der Unsterblichen und die Veredelung, die eher als geistiger denn als rein materieller Prozess zu verstehen ist. Mit Nachdruck weist er darauf hin, dass die Thematik der Unsterblichkeit von vielen Taoisten völlig missverstanden wird, und dass Unsterblichkeit als geistiges Stadium zu verstehen ist, das nur der geistig Aufgeschlossene erreichen kann!

T'ao Hung-Ching (2. Hälfte des 5. Jh.) soll schon als Zehnjähriger die Schriften des Pao P'u Tzu mit Eifer studiert haben und bei der Lektüre zum Entschluss gekommen sein, seinen Körper nach den taoistischen Regeln zu nähren, um sich ein hohes Alter zu versichern. Im Alter von 85 Jahren glich T'ao Hung-Ching noch so sehr einem Jüngling, dass ihn die darüber erstaunten Leute baten, das Geheimnis unvergänglicher Jugendfrische preiszugeben. Zuweilen wurde er dabei beobachtet, wie er in einem von einer Quelle gespeisten Felsbecken badete. Wegen seiner bis ins hohe Alter anmutigen und jugendlichen Erscheinung wurde er immer wieder für den Quellgeist gehalten!

In zahlreichen überlieferten Anekdoten und Legenden kommen die unterschiedlichsten Vorstellungen von Unsterblichen zum Ausdruck, die vom Grotesken und Phantastischen bis zum Erhabenen reichen. Überall, wo chinesische Kunstgegenstände aufgestellt sind, finden sich die Darstellungen der „Acht Unsterblichen": Einige sind anmutig anzusehen: Han Hsiang-Tzu ( Abb. 3: Erster von links) ist ein jugendlich aussehender Unsterblicher, dessen Zuneigung der Musik und Dichtung galt. In einem Gedicht, das er verfasst hatte, beschreibt er das Glück, in einer von einem nebelumwobenen Sturzbach verborgenen Grotte zu leben, Mitternachtstau zu schlürfen, bei Sonnenaufgang von rosafarbenen Wolken zu frühstücken und Perlen durch die Kraft der Musik zu schmelzen. Lan Ts'ai-Ho ( $\bullet$ Abb. 3: Fünfter von links) wird als Jüngling von rosiger Gesichtsfarbe dargestellt, der sein Haar in zwei seitlichen Knoten trägt und von Unverständigen oft für ein Mädchen gehalten wird. Seine jugendliche Erscheinung erinnert daran, dass er im Besitz des Geheimnisses der sich unablässig erneuernden Jugend ist. Ho Hsien-Ku 
( Abb. 3: Im Vordergrund rechts) ist die einzige weibliche Gestalt in der Runde. Nachdem sie als Kind Glimmerpulver eingenommen und die Unsterblichkeit erlangt hatte, legte sie ein Keuschheitsgelübde ab. Auf die Frage, ob sie unter den übrigen, männlichen Unsterblichen, nicht weibliche Gesellschaft vermisse, gab sie zur Antwort, dass die Kameraden, die das Tao gefunden hatten, die Eigenschaften beider Geschlechter besäßen. Andere wirken würdevoll, und mindestens einer erscheint auf humorvolle Weise grotesk. Eine weitere Gruppe von Unsterblichen, die Kennern der chinesischen Kunst vertraut sind, besteht aus Gestalten, welche die drei fundamentalen Wünsche des breiten Volkes darstellen: Fu (Reichtum), $\mathrm{Lu}$ (gesellschaftlicher Rang) und Shou (langes Leben). Insbesondere der ehrwürdige Shou stellt die Verkörperung des taoistischen Ideals dar, das langes Leben und Unsterblichkeit praktisch synonym gebraucht, aber Reichtum und gesellschaftlichen Rang wie zwei Flüche, die auf den Menschen lasten, behandelt. Shou ist an Symbolen für Langlebigkeit zu erkennen: einem außergewöhnlich hohen, unbehaarten Schädel, einem Stab aus Pfirsichholz und einem gefleckten Hirsch, der sich an ihn schmiegt.

Seit frühster Zeit war die Suche nach Drogen verbreitet, die die Kraft besaßen, die Jugend zu verlängern, Alte zu verjüngen und Langlebigkeit zu fördern. Obwohl zu den Inhaltsstoffen der Elixiere, die man mischte, auch Gifte gehörten, leisteten die Taoisten einen großen Beitrag zur Entwicklung der TCM. Alte taoistische Rezepte nennen immer wieder eine „rote Perle“ oder „Pillen-Perle“, wobei das chinesische Schriftzeichen für „rot“ auch „Pille“ bedeutet und sich wahrscheinlich von Zinnober ableitet. Das Pen Ts'ao (die „Grosse Pharmakopöe“) erklärt:

„Die Menschen haben seit langem Zinnober genommen, um ihre Kräfte zu mehren, ihre Jugend zu bewahren und ihre Körper leicht zu machen."

Die Liste der Zutaten für das magische Elixier enthielt neben den „fünf Mineralien“ Zinnober („roter Sand“), Schwefelarsen („Hahnengelb“), Glimmer („Wolkenmutter“), Quarz („Mineralblumen“) und verschiedene Arten von Stalaktiten („Liebesmilch“), auch Gold, Silber, verschiedene Arten der Pflanze chih, Jade und Perlen. Bei der Herstellung ihrer Elixiere waren die taoistischen Alchimisten erfüllt von einer Ehrfurcht vor vielfarbigen Flammen und Dämpfen, die ein Gefühl des Mysteriums vermittelten. Zugleich setzten sie sich aber auch der scharfen Kritik und dem Spott ihrer überlegteren Zeitgenossen aus:

„Der Gebrauch von Pillen anstelle des eigenen Körpers erfordert zu ihrer Herstellung fünf Metalle, acht Mineralien, Zinnober und Quecksilber! Der wahre Weg ist die formlose Vereinigung mit der Leere. Die 'neunfach geläuterte Pille' bedeutet in Wirklichkeit die Vereinigung mit der Leere. Die Grundsubstanz der Pille ist das Wesen der wahren Leere, die Mutter aller grossen Mittel.“

„Anhänger des Weges, die ihr nach Unsterblichkeit verlangt: Ihr könnt die 'neunfach geläuterte Pille' essen, wann ihr wollt - und die Folgen tragen!“

Hier wird deutlich, dass Gesundheit und langes Leben von denen erreicht wird, die in stiller Meditation mit dem grenzenlosen Tao kommunizieren, aber nicht jenen, die Pillen schlucken. Zudem war wohlbekannt, dass die Drogen gefährlich waren. Viele Alchimisten und ihre Kunden starben in der Tat an Blei- und Arsenvergiftungen. In der T'ang-Dynastie (618-907), als der Taoismus die Gunst des Kaiserhofes genoss, befanden sich unter den Opfern auch mehrere Kaiser!

Mit dem Niedergang der T'ang-Dynastie verlor der Taoismus Einfluss am Hof, und wurde durch den Konfuzianismus als Staatskult ersetzt, der vor allem während der Sung- (960 - 1279)

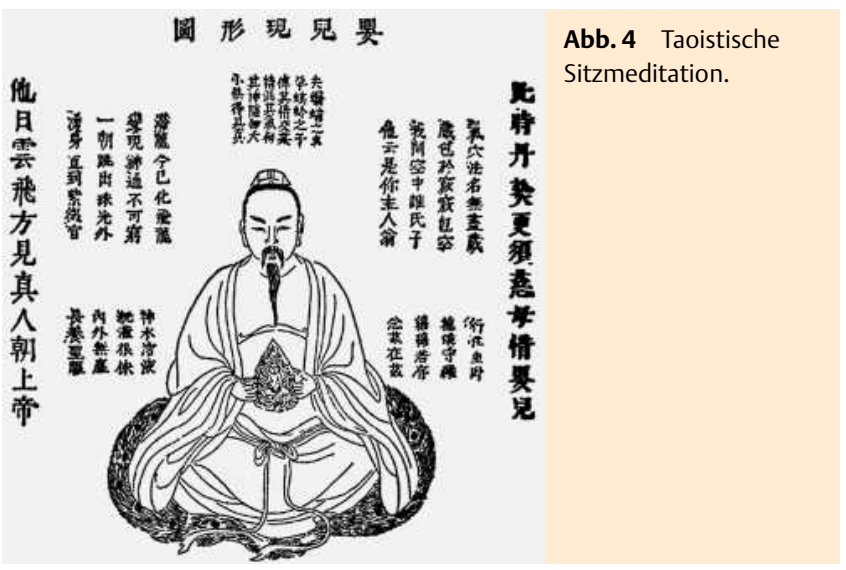

und der Ming-Dynastie (1368-1644) eine nachhaltige Wiederbelebung erfuhr. Dazwischen lag die Zeit der Mongolenherrschaft (Yuan-Dynastie, 1279-1368), in der vor allem der Buddhismus gefördert wurde. Allmählich wurden die taoistischen Lehren und Praktiken vom ch'an-Buddhismus aufgenommen, aus dem sich in Japan der Zen entwickelt hat. Dennoch fügte der Ming-zeitliche Gelehrte Wang Yang-Ming (1472-1528) dem konfuzianischen Ideal des „Edlen Menschen“ Eigenschaften hinzu, die bisher für den taoistischen Unsterblichen typisch waren. Während der Konfuzianismus bis dahin den Weisen als eine Person angesehen hatte, die sich mit vornehmer Sorge um das Wohlergehen der Gesellschaft kümmerte, wurde dieses Ideal um die Vorstellung erweitert, nach der die Vervollkommnung nur durch Konzentration aller körperlichen und geistigen Energien und durch Erkenntnis der wahren Natur des Menschen erlangt werden könnte. Der taoistische Begriff des ch'i (,Lebenskraft“) kam in Gebrauch, die Konfuzianer übernahmen die Lehre von der Einheit des Menschen mit der Natur und die Praxis der Sitzmeditation ( $\bullet$ Abb. 4). Der Lehre Wang Yang-Mings zufolge besitzt der Mensch im Innern einen Funken unbefleckten Geistes, der ihn mit dem Himmel vereint und den Weisen und Göttern potenziell ebenbürtig macht. Obschon er an sich rein ist, weil er genauso wenig verdorben werden kann wie ein Spiegel von dem, was er spiegelt, beschmutzt wird, sei dieser Spiegel aber von den Schleiern der Leidenschaften und Begierde, vom "Staub der zehntausend Dinge“ so dick überlagert, dass sich gewöhnliche Menschen ihr ganzes Leben lang dieses, ihres kostbarsten Besitzes, nicht bewusst würden. Daher sei es nutzlos, nach einem Schatz außerhalb des eigenen Geistes zu suchen; vielmehr scheine es geboten, den in den hintersten Winkeln verborgenen Schatz wieder zu entdecken, den Staub abzuwischen, ihn zu polieren und gänzlich im Schein seines Lichts zu leben. Diese Vorstellung führte dazu, bei den Meditationssitzungen mit Vorliebe eine Technik zu benutzen, bei der die Aufmerksamkeit auf einen Punkt zu lenken ist, der sich etwas hinter dem „Dritten Auge“ befindet. Wenn von diesem Punkt Licht ausströmt, das so deutlich sichtbar ist wie eine leuchtende Flamme vor den Augen, dann galt dies als Zeichen erfolgreicher Meditation. Auf diese Weise ergab sich, dass einiges aus dem Taoismus zunächst im ch'an-Buddhismus, dann aber auch im institutionalisierten Konfuzianismus fortleben konnte.

Während der Mandschu-Herrschaft während der Ch'ing-Dynastie (1644 - 1911) erfuhr der Taoismus keine wesentliche Weiterentwicklung. Die darauf folgende republikanische Regierung (1912 - 1948) des christlichen Generals Chiang Kai-Shek zeigte sich zwar gegenüber dem Buddhismus und Taoismus gleicher- 
maßen kopfscheu, aber erst der Kommunismus unter Mao TseTung (seit 1949) räumte in China der spirituellen Kultivierung endgültig keinen Platz mehr ein. Wie sehr der Glaube an die Wirksamkeit von Verjüngungsdrogen bis in unser Jahrhundert trotzdem fortbestanden hat, kann einer Meldung entnommen werden, die noch 1939 in der Ta Kung Pao, einer führenden Zeitung Chinas, erschien:

„Ein Herr aus Wan Hsien in der Provinz Szechuan, gebürtig im letzten Herrschaftsjahr des Kaisers Ch'ien Lung (1796), arbeitete während der letzten Jahre des folgenden Herrschers als Sekretär bei den Militärbehörden der Jangtsekiang-Region. Nach seiner Pensionierung reiste er nach Tibet, um Heilkräuter zu suchen, und bliebe so lange verschwunden, dass man ihn für verschollen hielt. Im Herbst 1931 kehrte er jedoch im Alter von 135 Jahren in seinen Heimatdistrikt zurück, wo ihn viele alte Einwohner als jemanden wieder erkannten, dem sie in ihrer frühen Jugend begegnet waren. Trotz seiner grauen Haare wirkte er nicht älter als 50 und hatte sich überhaupt kaum verändert.“

\section{Abstract}

\section{The Way of Longevity}

Medicine belongs to the most ancient achievements of human culture. Over millenia and through all cultures medicine has accompanied mankind, since it represents the response to an elementary awareness and dread of the unexpected interruption of well-being, the pain of physical ailment, and the horror of death. Especially old age as the final pathway of life has preoccupied and driven philosophers, scientists and physicians to seek the conditions underlying the aging process, age-related disorders, and ultimately death, with the aim to prevent or avert them. Going over the history of early Western medicine reveals an interconnection of medicine with culture, religion, and philoso- phy, until in the $19^{\text {th }}$ century there occurred a reduction of dietetics to diet, of hygiene to technology, and of the concept of health to measurable dimensions, while such basics as accord in lifestyle, striking the right balance, relaxation, and attempt for harmony have been neglected. In contrast, meditation, relaxation exercises, change between peace and motion, esthetic recuperation, and religious absorption have remained important elements of the Eastern doctrines of medicine, of Ayurveda and Traditional Chinese Medicine. The results of these methods can be explained by extraordinary psychophysical effects, which modern Western medical research and anti-aging medicine have just begun to acknowledge again.

\section{Literatur}

1 Trüeb RM. Die Jagd nach dem Jungbrunnen. Akt Dermatol 2007; 33: $297-309$

2 Blofeld J. Taoism - the Quest for Immortality. London: Allen \& Unwin, 1979

3 Der beste Weg unter dem Himmel. Sexuelle Körpertechniken aus dem alten China. Zwei Bambustexte aus Mawangdui. Übersetzt und kommentiert durch Pfister Rudolf. Museum Rietberg, 2003

4 Hackl M. Jung und schön mit Hui Chun Gong. Die geheimen Verjüngungsübungen der chinesischen Kaiser. Kreuzlingen: Irisiana, 2002

$5 \mathrm{Nan} \mathrm{H-C.} \mathrm{Tao} \mathrm{\&} \mathrm{Longevity.} \mathrm{Mind-Body} \mathrm{Transformation.} \mathrm{York} \mathrm{Beach:}$ Samuel Weiser, Inc, 1984

6 Pirk K. Den Alterungsprozess umkehren. Das Lebenselixier des Maharishi Ayur-veda. Bielefeld: J. Kamphausen, 2001

7 Schott H (Hrsg). Meilensteine der Medizin. Dortmund: Harenberg, 1996

8 Toellner R (Hrsg). Illustrierte Geschichte der Medizin. Salzburg: Andreas \& Andreas, 1986

9 Trüeb RM. Anti-Aging. Von der Antike zur Moderne. Darmstadt: Steinkopff, 2006

10 Wilhelm R, Jung CG. Geheimnis der Goldenen Blüte. Das Buch von Bewusstsein und Leben. 7. Auflage. München: Diederichs Gelbe Reihe, 1996

11 Darga M (Hrsg). Xingming Guizhi. Das alchemistische Buch von innerem Wesen und Lebensenergie. München: Diederichs Gelbe Reihe, 1999 